\title{
Smoke Deposition Velocity in Industrial Fire Environments
}

\author{
JEFFREY S. NEWMAN, PAUL SU, and GEARY G. YEE \\ FM Global \\ 1151 Boston-Providence Turnpike \\ Norwood, Massachusetts 02062 USA
}

\begin{abstract}
Smoke deposition velocities, measured in a $1.0 \mathrm{~m}^{3}$ smoke exposure chamber, have been related to the decay in local smoke exposure concentrations as well as the smoke particle characteristics. These velocities, on the order of $10^{-4} \mathrm{~m} / \mathrm{s}$, are consistent with previous experimental work in a large-scale $1200 \mathrm{~m}^{3}$ enclosure. Calculated deposition velocities are in reasonable agreement with measured values, but tend to be underpredicted, most likely due to cloud settling effects associated with the smoke layer movement.
\end{abstract}

KEYWORDS: smoke damage, smoke deposition, industrial fires.

\section{INTRODUCTION}

The deposition of smoke resulting from the transport of combustion products away from the origin of a fire is a major source of damage in many industrial applications such as in facilities associated with semiconductor fabrication, data storage or processing and pharmaceutical manufacturing. Even in warehouse facilities with adequate fire protection, the damage associated with smoke deposition can far exceed that due to heat and water destruction.

The process by which smoke can deposit on various surfaces is often complex and can result from either a single dominant physical mechanism or a combination of mechanisms including particle inertia, sedimentation or gravitational settling, Brownian diffusion, thermophoresis or thermodiffusion, and electrostatic precipitation [1]. For electrically neutral aerosols, the governing mode of smoke deposition is primarily dependent on whether the transport flow is laminar or turbulent and the particle size.

The flow regime for smoke particle motion can be characterized by the magnitude of the particle Reynolds number, defining $\rho_{g}$ as the gas density, $d_{p}$ the particle diameter, $V$ the particle velocity and $\eta$ the gas viscosity, i.e.,

$\operatorname{Re}_{p}=\frac{\rho_{g} d_{p} V}{\eta}$

\section{Laminar Flow $\left(\operatorname{Re}_{p}<1\right)$ :}

1) For smoke particles $>1 \mu \mathrm{m}$ in diameter, the deposition is primarily due to gravitational settling. Stokes' law applies to particle motion when inertial forces are negligible compared with viscous forces, and gives the particle terminal settling velocity, $V_{T S}$ as

$V_{T S}=\frac{\rho_{p} d_{p}^{2} g}{18 \eta}$

where $g$ is gravitational acceleration.

2) For smoke particles $<1 \mu \mathrm{m}$ in diameter, both Brownian diffusion as well as gravitational settling are important deposition mechanisms. An important assumption in Eq. 2 is that the relative velocity of the gas at the surface of the particle is zero, a condition which is not met for particle sizes that approach the mean free path of the gas. The particles move faster than predicted by Stokes' law and a slip correction factor, $C_{c}$, is needed [2]: 
$C_{c}=1+\frac{2.52 \lambda}{d_{p}}$

where $\lambda$ is mean free path, which is $0.066 \mu \mathrm{m}$ for air at $1 \mathrm{~atm}$ and $20^{\circ} \mathrm{C}$. The slip correction factor is a multiplicative factor applied to the right-hand side of Eq. 2, increasing the terminal settling velocity.

\section{Turbulent Flow $\left(\operatorname{Re}_{p}>1\right)$ :}

1) For smoke particles $>1 \mu \mathrm{m}$ in diameter, the deposition begins to be influenced by both particle inertia as well as viscous effects, which was first shown by Friedlander and Johnstone in 1957 [3]. Inertial deposition occurs when a particle near a wall (surface) in the turbulent region is given sufficient lateral velocity toward the wall to be transported through the laminar layer and deposit on the wall (surface). The particle settling velocity transitions from a $d_{p}^{2}$ dependence in the Stokes regime to a $d_{p}^{1 / 2}$ dependence in the Newton drag regime. This transition can be accounted for by the introduction of a drag coefficient, $C_{D}$, in the calculation of settling velocity, i.e.,

$V_{T S}=\left[\frac{4 \rho_{p} d_{p} g}{3 C_{D} \rho_{g}}\right]^{1 / 2}$

Figure 1, for example, illustrates the functional relationship between $C_{D}$ and $\operatorname{Re}_{p}$ for spherical particles.

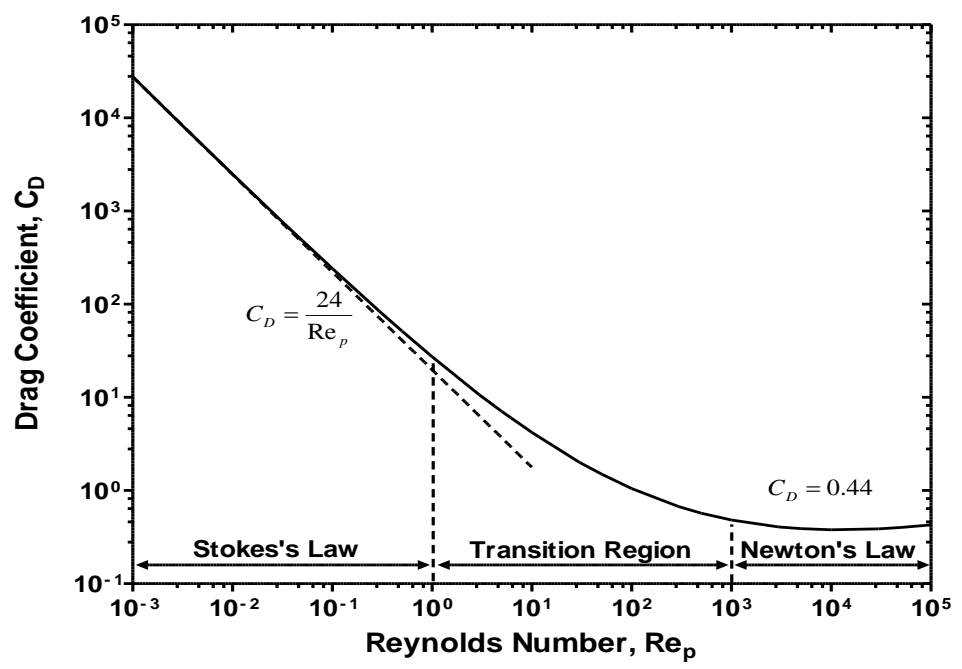

Fig. 1. Drag coefficient versus particle Reynolds number.

Numerous correlations have been developed to describe the drag coefficient and Reynolds number functional relationship. A particularly useful formulation covers the transition region $\left(1<\operatorname{Re}_{p}<1000\right)[1]$ :

$C_{D}=\frac{24}{\operatorname{Re}_{p}}\left(1+0.15 \operatorname{Re}_{p}^{0.687}\right)$

2) For smoke particles $<1 \mu \mathrm{m}$ in diameter, Brownian diffusion becomes important. The diffusion coefficient of an aerosol/smoke particle can be expressed in terms of particle properties by the StokesEinstein derivation [4]. In this derivation, the diffusion force on the particles, which causes the particles' net motion along the concentration gradient, is equated to the force exerted by the gas resisting the particles' motion, i.e., 


$$
D=\frac{k T C_{c}}{3 \pi \eta d}
$$

where $D$ is the particles' diffusion coefficient, $k$ is Boltzmann's constant and $T$ is the absolute temperature.

Thermophoresis can be important where large temperature gradients exist, e.g., from a smoke hot layer to a cold surface such as a wall or ceiling in an enclosure. Thermophoresis is the aerosol particle movement that results from the force exerted by a temperature gradient in a gas, producing a flow in the direction of decreasing temperature. This would be the dominant mechanism for very small particles $<0.1 \mu \mathrm{m}$, even at small gradients of $1{ }^{\circ} \mathrm{C} / \mathrm{cm}[1]$.

In addition, another phenomenon can occur at relatively high smoke concentrations. Cloud settling or mass subsidence occurs when the smoke concentration is sufficient to cause the entire cloud to move as an entity at a velocity significantly greater than the individual particle settling velocity [5]. (An aerosol or smoke cloud is defined as a region of high smoke concentration having a definite boundary in a much larger region of smoke-free air.) The difference in density causes the bulk motion of the smoke particles.

\section{EXPERIMENTAL SET-UP}

Figure 2 shows the schematic of the apparatus developed to measure the smoke deposition velocity resulting from the combustion of various materials. The combustion apparatus consisted of a tube furnace

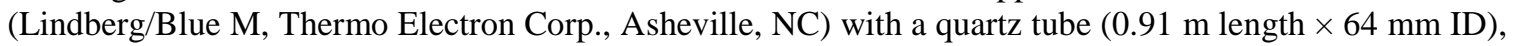
a flow monitor (Model Nos. HFM-300 \& THPS-100, Teledyne Hastings, Hampton, VA) and an igniter (FranceFormer unit, Scott Fetzer Co., Fairview, TN).

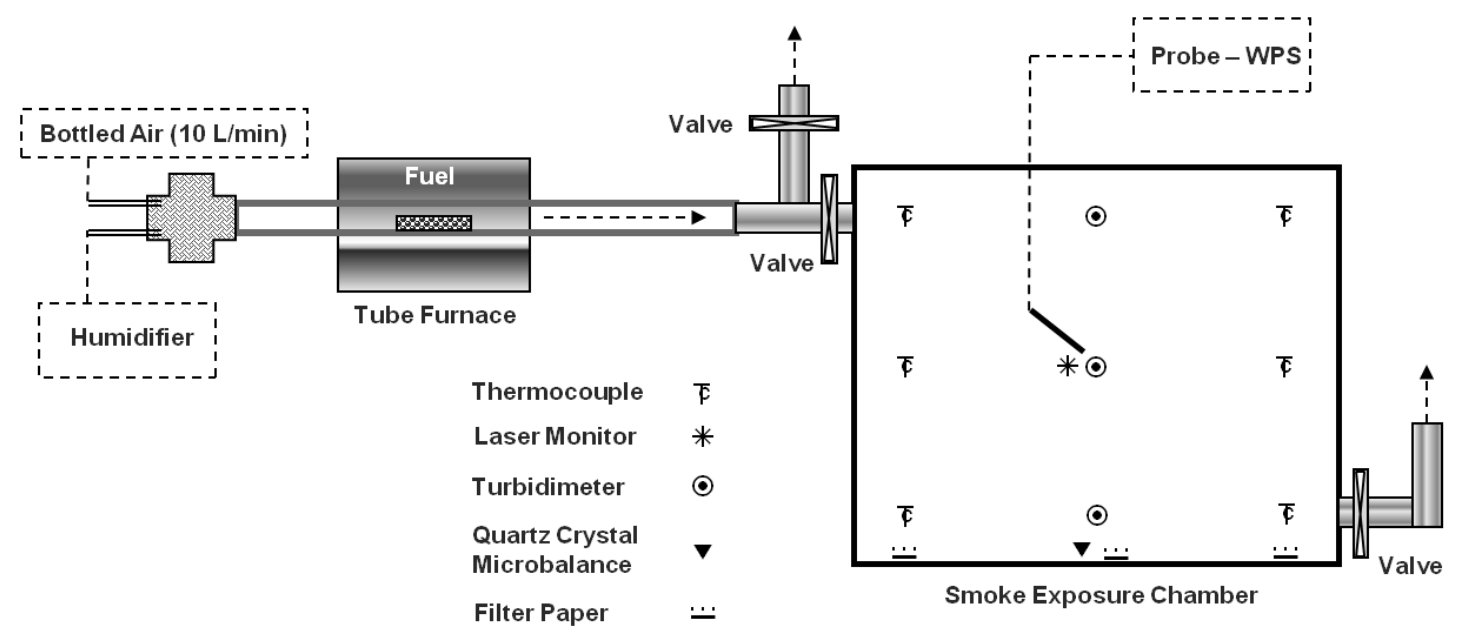

Fig. 2. Schematic of the experimental set-up for smoke damage characterization.

The smoke exposure chamber (SEC) has a volume of $1.0 \mathrm{~m}^{3}$, with dimensions of $0.91 \times 1.22 \times 0.91 \mathrm{~m}$ high, and was constructed of an aluminum frame with polymethylmethacrylate (PMMA) sides. Two ports (19 mm ID) with valves located on opposite sides were for the entry and exit of smoke through the chamber. Six thermocouples (Type K, Model No. KMQSS-062E-18, Omega Engineering Co., Stamford, $\mathrm{CT}$ ) were mounted in the chamber to monitor the temperature throughout the test. A laser (HeNe laser, $1 \mathrm{~mW}$ at $632.8 \mathrm{~nm}$, Model No. 05-STP-910-249, Melles Griot, Carlsbad, CA), mounted on the chamber ( $0.1 \mathrm{~m}$ from top of chamber, angling downward to $0.1 \mathrm{~m}$ up from the bottom opposite side), was used to measure the smoke concentration. In addition, three turbidimeters [6], operating on the same wavelength as the laser $(632.8 \mathrm{~nm})$, were mounted centrally $(0.1 \mathrm{~m}$ from top, mid-plane, and $0.1 \mathrm{~m}$ from bottom) on one side to monitor the level of smoke. The data acquisition (for the thermocouples, laser, and turbidimeters) consisted of Agilent Technologies (Model No. 34970A \& Agilent Benchlink Data Logger, version 1.4.000512, Santa Clara, CA) and National Instruments (Measurement and Automation Explorer, version 3.1.1.3004, NI-488.2, Austin, TX) systems. 
Three materials representative of facilities associated with semiconductor fabrication, data storage/processing and pharmaceutical manufacturing were selected for combustion evaluation: polycarbonate, polyvinyl chloride (PVC) and nylon. The polymers were in either powder form, rod-like form (1.6 mm diameter $\times 3.2 \mathrm{~mm}$ length or $6.4 \mathrm{~mm}$ diameter $\times 6.4 \mathrm{~mm}$ length $)$, or cut into small pieces $(2.4 \mathrm{~mm} \times 9.5 \mathrm{~mm})$. For each combustion test run, $12 \mathrm{~g}$ of polymer material (loaded onto a $25 \mathrm{~mm} \times$ $100 \mathrm{~mm}$ steel pan) was used with an air flow of $10 \mathrm{l} / \mathrm{min}$. The samples were ignited in the quartz tube at the tube furnace temperature range of $450-550^{\circ} \mathrm{C}$.

Conditions were selected such that smoke generated by combustion from the tube furnace could be rapidly captured in the exposure chamber by allowing the smoke to enter through a valve on one of the short sides of the chamber $0.1 \mathrm{~m}$ down from the top (shown at the top left of the SEC in Fig. 2). Air, as it was displaced by the entering smoke, was allowed to freely exhaust from the chamber through a similar valve in the opposite end panel $0.1 \mathrm{~m}$ up from the bottom of the chamber. All tests were conducted under flaming combustion conditions, with smoke yields for the selected materials expected to be comparable to those obtained by the use of the fire propagation apparatus $[7,8]$. The initial relative humidity in the chamber was controlled to $50 \pm 10 \%$.

Typically, smoke was generated for five to ten minutes (i.e., time for complete combustion of the sample) after which time the chamber was sealed by closing the entrance and exit valves. Smoke settling measurements were then continued for approximately one hour. A quartz crystal microbalance (QCM200 Digital Controller, QCM25 $5 \mathrm{MHz}$ crystal oscillator with $25 \mathrm{~mm}$ diameter quartz crystals, Stanford Research Systems Inc., Sunnyvale, CA) was placed at the chamber bottom surface center. In addition, $90 \mathrm{~mm}$ diameter Teflon filter media targets were located at the base of the chamber, at the center and nominally $0.25 \mathrm{~m}$ diagonally from each corner, to supplement the QCM measurements by measuring the total smoke mass deposited during the course of the hour-long monitoring experiment. Smoke particle size was measured using a wide-range particle spectrometer (WPS Model 1000XP, Range - $10 \mathrm{~nm}$ to $10000 \mathrm{~nm}$, MSP Corporation, Shoreview, MN), which consisted of a differential mobility analyzer/condensation particle counter and a laser light scattering particle spectrometer. This instrument

was connected, via Teflon tubing, to a $12.7 \mathrm{~mm}$ inner diameter stainless steel probe that sampled smoke from the center of the chamber.

\section{SMOKE DEPOSITION FROM POLYCARBONATE}

Polycarbonate was selected as the baseline fuel/smoke source for the smoke decay/deposition characterization measurements. The tube furnace was typically charged with $12 \mathrm{~g}$ of polycarbonate beads on a pan and the fuel was ignited with a spark igniter. As described above, the resulting smoke was quickly captured in the smoke exposure chamber and allowed to dissipate/decay over a period of about one hour. Figure 3a illustrates the smoke concentration distribution history using data obtained from the laser extinction measurements as well as the three smoke turbidimeters. The smoke concentration appears to decay rapidly at the very top of the chamber and remain suspended in the middle to the bottom of the chamber, which is consistent with visual observations. Figure $3 \mathrm{~b}$ also presents the middle turbidimeter data plus laser data and indicates similar concentration trends from the two sets of measurements. Preceding tests used the laser measurements to characterize the average change in smoke concentration, while the data shown in Fig. 3a were taken to assess whether the smoke stratification was significant within the smoke exposure chamber. Figure 4a plots the gas temperature rise above ambient recorded at the same vertical locations as the turbidimeter measurements. Essentially, the smoke within the chamber was "cold" smoke with about $0.5{ }^{\circ} \mathrm{C}$ temperature rise above ambient (or excess gas temperature) and about $0.25{ }^{\circ} \mathrm{C}$ initial differential between the top and bottom of the chamber. From Figs. 3a and 4a, the decay in smoke concentration and excess gas temperature appear to trend together. 


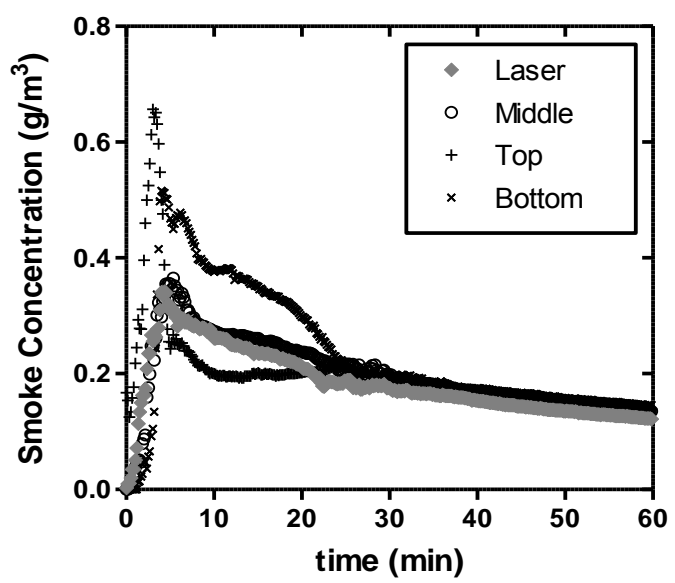

(a)

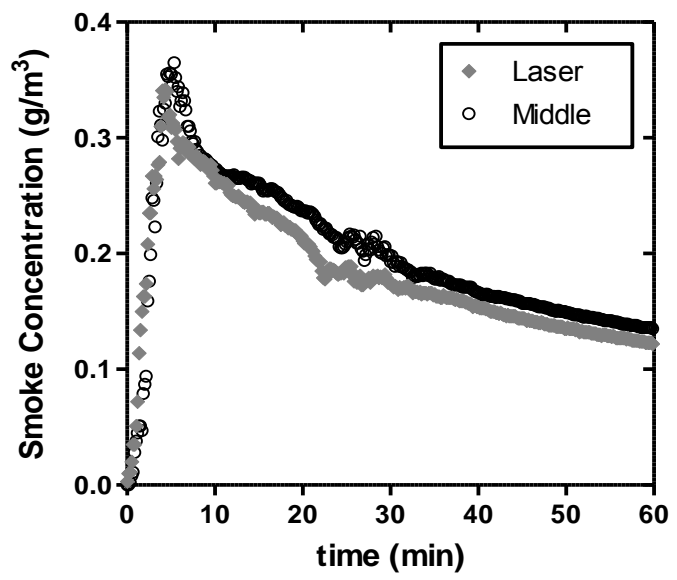

(b)

Fig. 3. Example test results for polycarbonate: (a) smoke distribution in smoke exposure chamber; (b) middle turbidimeter and laser measurements.

Figure $4 \mathrm{~b}$ plots an example of the normalized smoke mass balance history for polycarbonate smoke in the smoke exposure chamber. The upper set of data in the figure tracks the smoke decay as determined by the laser measurements in the chamber. The data were normalized by taking the instantaneous smoke concentration data (see Fig. 3b), multiplying by the total volume of the smoke exposure chamber $\left(1.02 \mathrm{~m}^{3}\right)$ and dividing by the peak smoke mass value from the laser measurement. The time is measured from the peak concentration. The lower set of data in the figure is from the deposition measurements using the QCM. The data were calculated by taking the instantaneous weight data (given as $\mathrm{g} / \mathrm{m}^{2}$ ) from the QCM, multiplying by the floor area of the smoke exposure chamber $\left(1.12 \mathrm{~m}^{2}\right)$ and dividing by the same peak smoke mass value as used for the smoke decay data. The data, as presented in Fig. 4b, result in a simple method to assess the mass balance in the chamber. The sum of the normalized smoke decay and normalized smoke deposition ideally should be equal to one at any time increment, assuming no additional losses to walls.

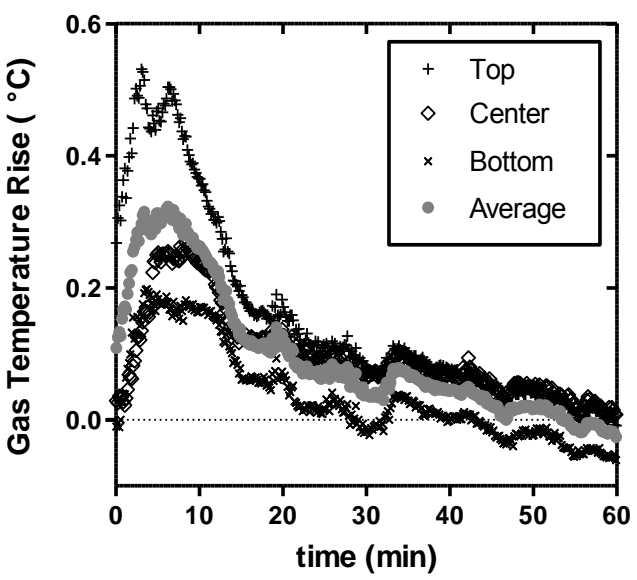

(a)

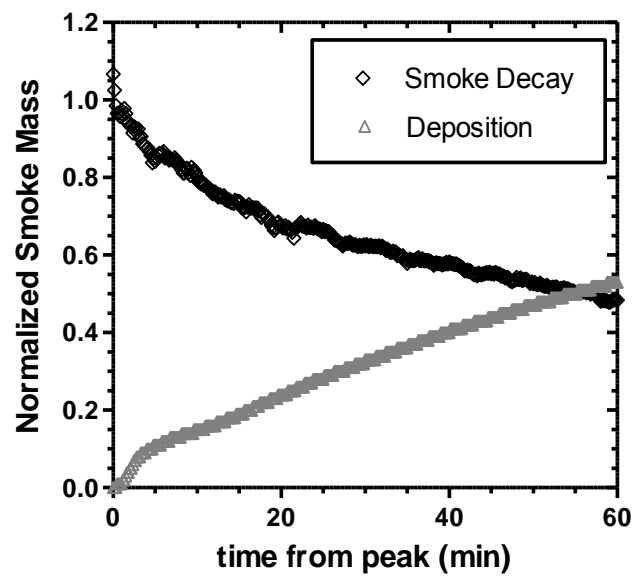

(b)

Fig. 4. Example test results for polycarbonate: (a) gas temperature distribution history;

(b) normalized smoke mass history.

A series of curve fit function analyses were performed on the smoke decay data as illustrated by Fig. 4b with the intent that the deposition data should be represented by a complementary function determined only by the curve fit to the decay data. Here, the two complementary functions are defined such that their sum should always yield a constant, i.e., a value of one. The decay process appears to be a two-phase 
exponential decay, where the overall process can be represented by the sum of a short relatively fast decay stage and a longer relatively slow decay stage. This overall process can be represented by the following:

Decay $=\left\{\%\right.$ Fast $* \exp \left(-t / \tau_{\text {fast }}\right)+(100-\%$ Fast $\left.) * \exp \left(-t / \tau_{\text {slow }}\right)\right\} / 100 \%$

where the term beginning with \%Fast is the portion of the overall process associated with the fast decay stage with time constant of $\tau_{\text {fast }}$, and the second term beginning with $(100-\%$ Fast $)$ is the portion associated with the slow decay stage and a time constant of $\tau_{\text {slow }}$. [Note that Eq. 7 has been normalized such that it scales between 0 and 1 consistent with the data representation as illustrated by Fig. 4b.] A number of experiments was performed to assess the impact of burn time (i.e., how quickly the polycarbonate was heated and combusted within the tube furnace leading to the smoke exposure chamber) on the smoke decay function represented by Eq. 7 .

The time constants were found to be essentially independent of the burn time (typically about $7 \mathrm{~min}$ ) with average values of $11 \pm 3 \mathrm{~min}$ and $110 \pm 20 \mathrm{~min}$ for the fast and slow decay time constants, respectively. The value of \%Fast was also nearly constant at around $25 \%$. Figure 5a plots Fig. $4 \mathrm{~b}$ with the line through the decay curve calculated using Eq. 7 with $\tau_{\text {fast }}=11 \mathrm{~min}$ and $\tau_{\text {slow }}=110 \mathrm{~min}$. The value of \%Fast is determined by the curve fit as $24 \%$. The line through the deposition data is determined by the complementary function to Eq. 7, i.e.,

$$
\text { Deposition }=\left\{\% \text { Fast } \times\left[1-\exp \left(-t / \tau_{\text {fast }}\right)\right]+(100-\% \text { Fast }) \times\left[1-\exp \left(-t / \tau_{\text {slow }}\right)\right]\right\} / 100 \%
$$

with the values of \%Fast, $\tau_{\text {fast }}$ and $\tau_{\text {fast }}$ determined by the decay curve fit described above. In Fig. 5a, as expected, the smoke decay mass data are well-fit using the two-phase exponential representation described by Eq. 7. The complementary deposition function trends well with the data, somewhat over predicting the smoke mass deposition during the middle portions of the smoke settling process within the smoke exposure chamber. A modified QCM crystal was developed to increase the collection area from $0.76 \mathrm{~mm}^{2}$ to $3.0 \mathrm{~cm}^{2}$ to assess whether the instrument response and sensitivity could be a factor in the measured deposition rates. Figure $5 \mathrm{~b}$ plots the normalized smoke mass history for polycarbonate using the modified QCM crystal. The larger area crystal proved to be highly unstable at low mass deposition and was essentially unresponsive until approximately $15 \mathrm{~min}$ after the peak (corresponding to a specific density of $\sim 5 \mu \mathrm{g} / \mathrm{cm}^{2}$ ). Subsequently, the crystal exhibited long period oscillations, perhaps due to some off center particle deposition. A highpass filter was applied to attenuate the low frequencies. The resulting filtered data are shown by the dark

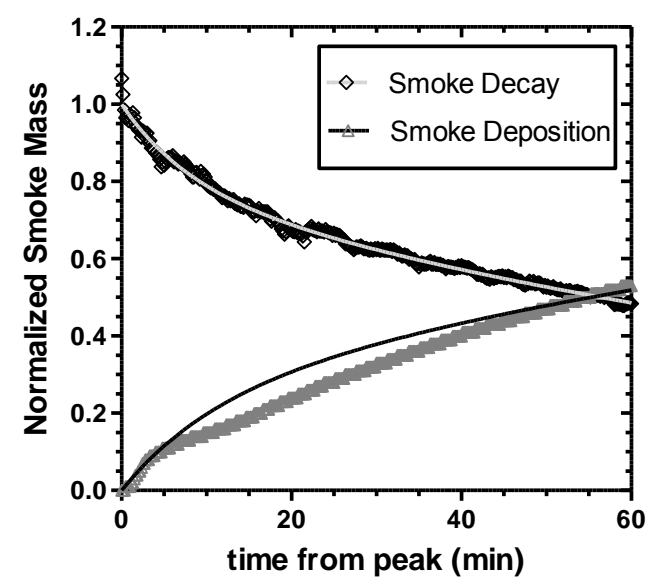

(a)

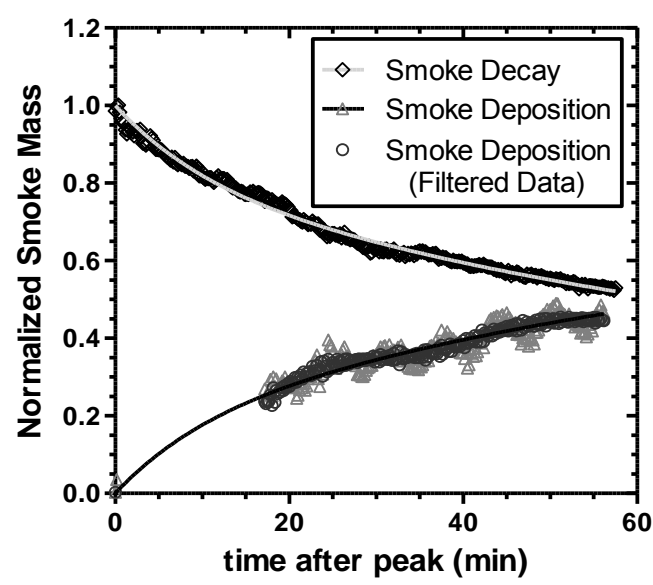

(b)

Fig. 5. Polycarbonate results: (a) smoke decay/deposition model; (b) normalized smoke mass history (large area QCM). 
gray open symbols in the figure. The deposition curve fit, shown by the lower curve, is unaffected by the filtering process and shows a better correlation with the deposition data at intermediate times.

Figure 6a gives the combined data from the original (small) and modified (large) QCM crystals. The solid lines in the figure are the calculated curve fits for the combined sets of data and show very good agreement with the measured values. Figure 6a is also reflective of the reproducibility of the experiments, typically $\pm 10 \%$. The mass balance error is also reflected in the figure and typically was also $\pm 10 \%$, but occasionally could reach $\pm 15 \%$, most likely due to non-uniformity in smoke deposition across the bottom of the smoke exposure chamber. The potential departure from uniform conditions was routinely checked by weighing the total mass deposition on Teflon filter targets distributed on the bottom of the chamber, with variations consistently less than $\pm 15 \%$ from the average. Subsequently, all deposition measurements were taken with the original, smaller area crystal, due to the instability problems (as well as sensitivity issues at lower deposition masses) with the modified larger area crystal.

Since the data for mass balances indicate that all the smoke is accounted for, the fit to the data is not required. Therefore, the following relationship holds:

$\int_{0}^{t} \dot{m}_{d}^{\prime \prime}(t) d t=\int_{0}^{t} V_{T S} C_{s}(t) d t$

and

$\dot{m}_{d}^{\prime \prime}(t)=V_{T S} C_{s}(t)$

where $\dot{m}_{d}^{\prime \prime}$ is the smoke deposition rate per unit surface area, $C_{s}$ is the smoke concentration and $V_{T S}$ is the terminal settling velocity. Therefore, the smoke deposition/settling velocity for the polycarbonate smoke can be ascertained by plotting the specific smoke mass deposition $\left(\mathrm{g} / \mathrm{m}^{2}\right)$ against the time integral of the smoke mass concentration $\left(\mathrm{g} \cdot \mathrm{s} / \mathrm{m}^{3}\right)$. The slope of the resulting curve yields $V_{T S}$, as given by Eq. $9 \mathrm{~b}$. Figure $6 \mathrm{~b}$ shows a value of $1.9 \times 10^{-4} \mathrm{~m} / \mathrm{s}$ for polycarbonate over the observed one-hour time frame.

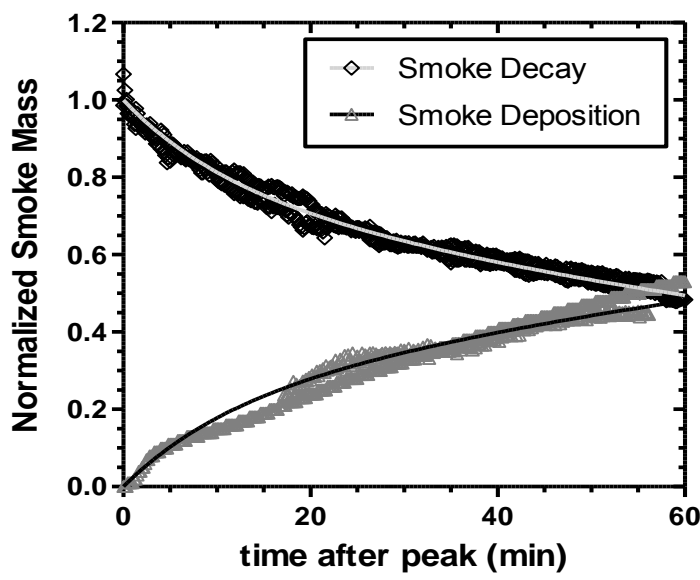

(a)

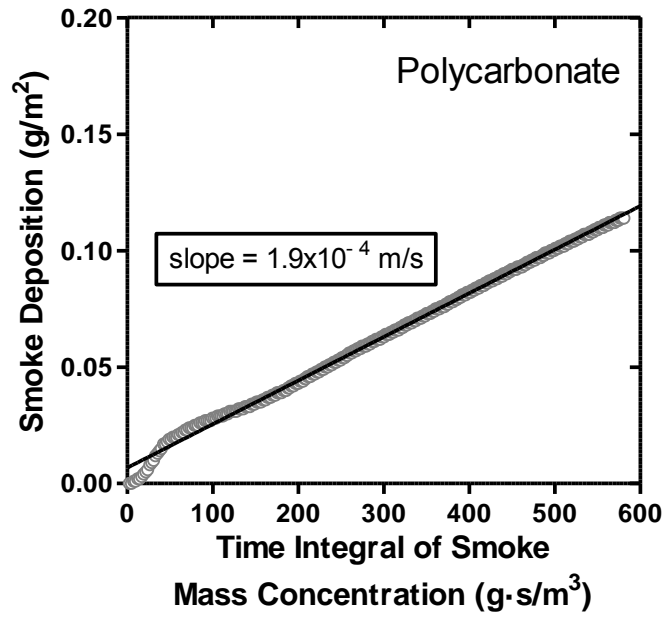

(b)

Fig. 6. Polycarbonate results: (a) normalized smoke mass history (combined QCM);

(b) smoke deposition velocity.

\section{SMOKE DEPOSITION FROM PVC}

PVC was selected as the second material to evaluate using the tube furnace/smoke exposure chamber apparatus. The procedures followed were virtually identical to those used previously for polycarbonate. The 
fuel used was $12 \mathrm{~g}$ of pure PVC powder (i.e., no added plasticizers or fillers), which was loaded into a tray using the conditions established during the polycarbonate benchmarking. The fuel was ignited as before with a spark igniter and the smoke exposure chamber was charged with the resulting PVC smoke during the duration of the combustion process ( $\sim 3 \mathrm{~min})$. Figure 7 a gives the smoke mass history, normalized as before with the peak smoke mass as measured by the laser directed diagonally across the chamber.

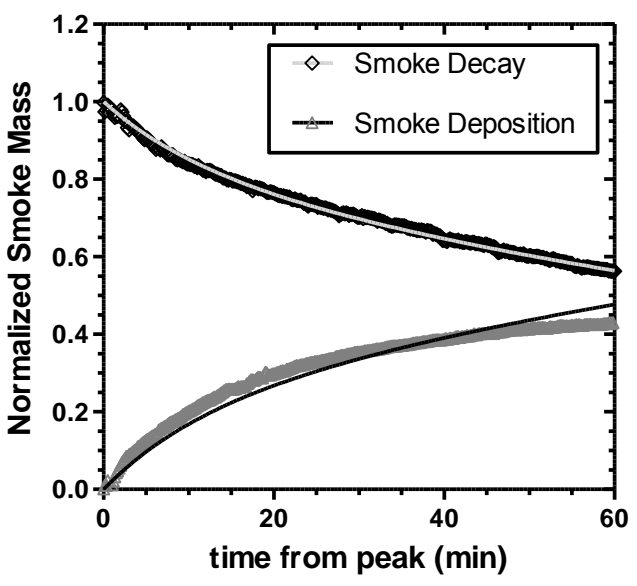

(a)

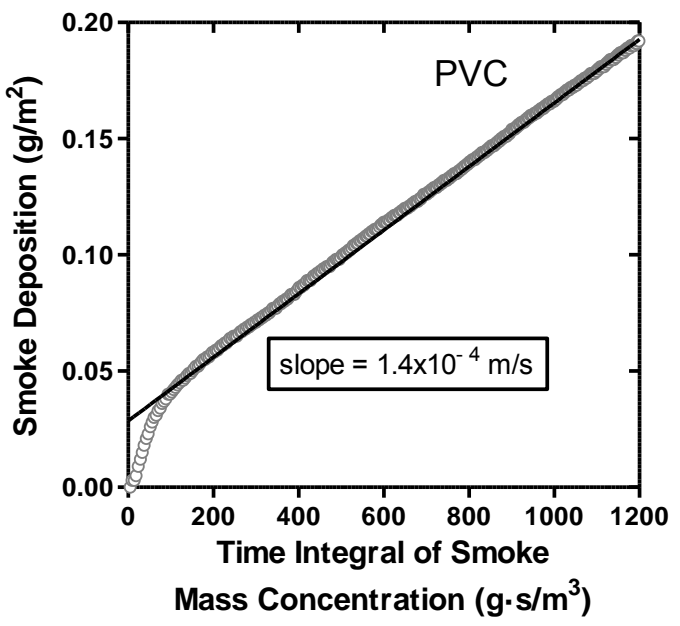

(b)

Fig. 7. PVC results: (a) normalized smoke mass history; (b) smoke deposition velocity.

The line curve fit for the decay data is from the application of Eq. 7 with $\tau_{\text {fast }}=11 \mathrm{~min}$ and $\tau_{\text {slow }}=110 \mathrm{~min}$ as previously used for polycarbonate. The value of \%Fast is determined by the curve fit as $20 \%$. Similarly, the complementary smoke deposition function, as shown in the lower black line in the figure, was calculated with Eq. 8 using the above values for $\tau_{\text {fast }}, \tau_{\text {slow }}$ and $\%$ Fast. The deposition data are again well fitted by modeling just the decay data. The cumulative mass balance error is about $-10 \%$, which is evident by the slight divergence shown in the figure between the idealized calculated and measured smoke mass deposition at the end of the 60 min observation period.

The smoke deposition velocity for PVC is given as the slope of the curve shown in Fig. 7b. The value of $1.4 \times 10^{-4} \mathrm{~m} / \mathrm{s}$ is comparable to the settling velocity previously calculated for polycarbonate $(1.9 \times$ $\left.10^{-4} \mathrm{~m} / \mathrm{s}\right)$. An initially higher deposition velocity is indicated by the data in the lower left in the figure, which was also observed to a lesser degree in the polycarbonate data (see Fig. 6b).

\section{SMOKE DEPOSITION FROM NYLON}

Nylon was selected as the third and final material to evaluate with the tube furnace/smoke exposure chamber apparatus. The specific fuel used was nylon-6, a semicrystalline polyamide made from a single monomer, and was selected as representative of the class of nylon polymers. Twelve grams of nylon were loaded into a tray using the conditions established during the polycarbonate benchmarking. The fuel was ignited as before with a spark igniter and the smoke exposure chamber was charged with the resulting nylon smoke during the duration of the combustion process $(\sim 4.5 \mathrm{~min})$. Similar to shown previously for polycarbonate and PVC, Fig. 8a gives the normalized smoke mass history for both the smoke decay and deposition of nylon in the smoke exposure chamber. The same values for $\tau_{\text {fast }}$ and $\tau_{\text {slow }}$ were used as previously for the decay curve fit in the figure $(11 \mathrm{~min}$ and $110 \mathrm{~min}$, respectively). The value for \%Fast was determined as $18 \%$. The complementary function for the smoke deposition was again calculated by Eq. 8 using the decay values for $\tau_{\text {fast }}, \tau_{\text {slow }}$ and $\%$ Fast. The cumulative mass balance calculation error is about $+5 \%$, as shown by the slight under prediction of the total mass deposition at the end of the observation period.

The smoke deposition velocity for nylon is given as the slope of the curve shown in Fig. 8b. The value of $2.5 \times 10^{-4} \mathrm{~m} / \mathrm{s}$ is the largest settling velocity of the three materials studied. An initially higher deposition 
velocity previously observed for polycarbonate (see Fig. 6b) and PVC (see Fig. 7b) is only slightly evident in the nylon data.

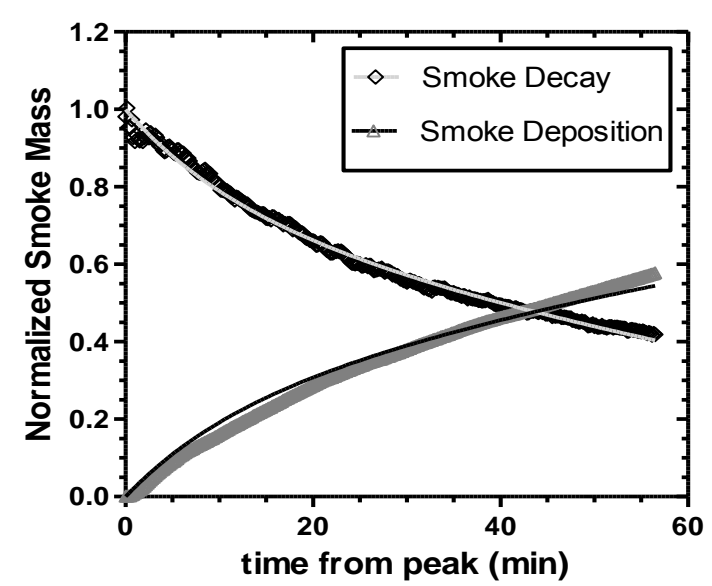

(a)

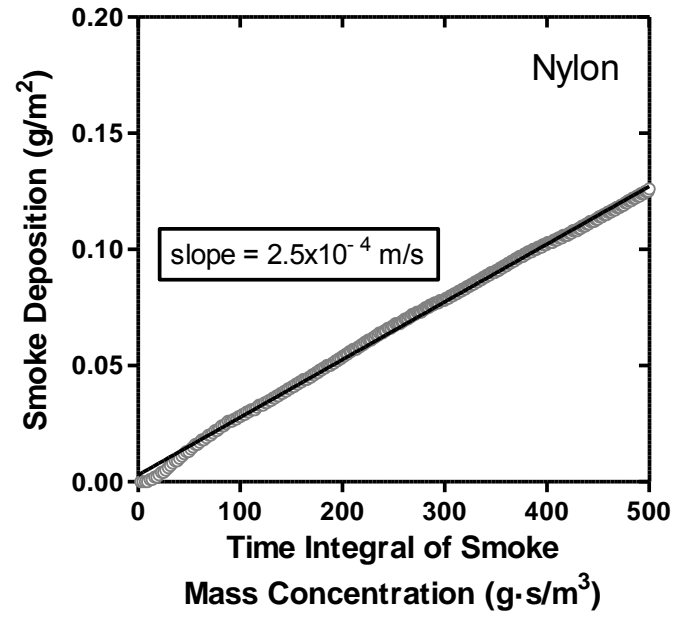

(b)

Fig. 8. Nylon results: (a) normalized smoke mass history; (b) smoke deposition velocity.

\section{PARTICLE SIZE MEASUREMENTS}

Smoke particle measurements were made using a wide-range particle spectrometer (WPS) [9]. The WPS is a high-resolution aerosol spectrometer capable of measuring the diameter and number concentration of aerosol particles. It consists of essentially two integrated instruments. A differential mobility analyzer (DMA) in combination with a condensation particle counter (CPC) is used to measure smaller particles between 10 and $500 \mathrm{~nm}$ in diameter. The DMA/CPC measures size based on particle mobility and has 96 channels of resolution. A laser light scattering particle spectrometer (LPS) measures particles in the 350 to $10,000 \mathrm{~nm}$ diameter range using an optical technique and has 24 channels of resolution. The WPS is calibrated with NIST traceable particle sizing with polystyrene latex spheres (e.g., 100, 269, 1361, and $7979 \mathrm{~nm}$ spheres).

The wide-range particle spectrometer determines particle characteristics on the basis of the aerodynamic equivalent diameter $\left(d_{a}\right)$, which is the diameter of a unit-density sphere having the same gravitational settling velocity as the measured particle. The aerodynamic equivalent diameter takes into account the shape, roughness, and aerodynamic drag of the particle [1]. For example, Stokes' law written in terms of $d_{a}$ and a standard particle density, $\rho_{0}\left(1000 \mathrm{~kg} / \mathrm{m}^{3}\right)$ gives the following for the terminal settling velocity, $V_{T S}$ in air:

$V_{T S}=\frac{\rho_{0} d_{a}^{2} g}{18 \eta}$

where $g$ is the acceleration due to gravity and $\eta$ is the viscosity of air. Particle size statistics are represented by the WPS software as a typical log-normal particle size distribution function, which has shown to be useful in characterizing smoke aerosols [1]. For this type of distribution, the log of the particle diameter follows a Gaussian distribution. Therefore, the geometric mean diameter, $d_{g}$, is given by:

$\ln d_{g}=\frac{\sum n_{i} \ln d_{i}}{N}$ 
where $n_{i}$ is the number of particles with diameter $d_{i}$ and $N$ is the total number of particles. The geometric standard deviation, $\sigma_{g}$, is given by:

$\ln \sigma_{g}=\left[\frac{\sum n_{i}\left(\ln d_{i}-\ln d_{g}\right)^{2}}{N-1}\right]^{1 / 2}$

The geometric standard deviation is dimensionless with a value equal to or greater than 1.0. One geometric standard deviation represents the range of particle sizes from $\left(d_{g} / \sigma_{g}\right)$ to $\left(d_{g} \times \sigma_{g}\right)$.

\section{Smoke Exposure Chamber Measurements}

The WPS was used to sample smoke for each of the three materials previously evaluated for smoke deposition in the SEC. Combustion products were again captured using the tube furnace as a generation source. Samples were taken at two-minute intervals from the center of the SEC, as illustrated previously in Fig. 2. Figure 9 presents particle size histories for polycarbonate, $\mathrm{PVC}$ and nylon. The particle size is given as the mass mean aerodynamic equivalent diameter, $\vec{a}_{a}$. Also included in the figure is the geometric standard deviation, $\sigma_{g}$, for each two-minute time slice plotted on the right ordinate. The straight lines in each of the plots shown in the figure represent the average values for each material after $20 \mathrm{~min}$ from the initiation of the smoke sampling, with a $\vec{d}_{a}$ of $3.0,1.1$ and $0.4 \mu \mathrm{m}$ for polycarbonate, PVC and nylon, respectively. $\sigma_{g}$ remained fairly constant for polycarbonate and nylon (3.4 and 2.0, respectively), while the distribution narrowed over time for PVC (1.8 on average).
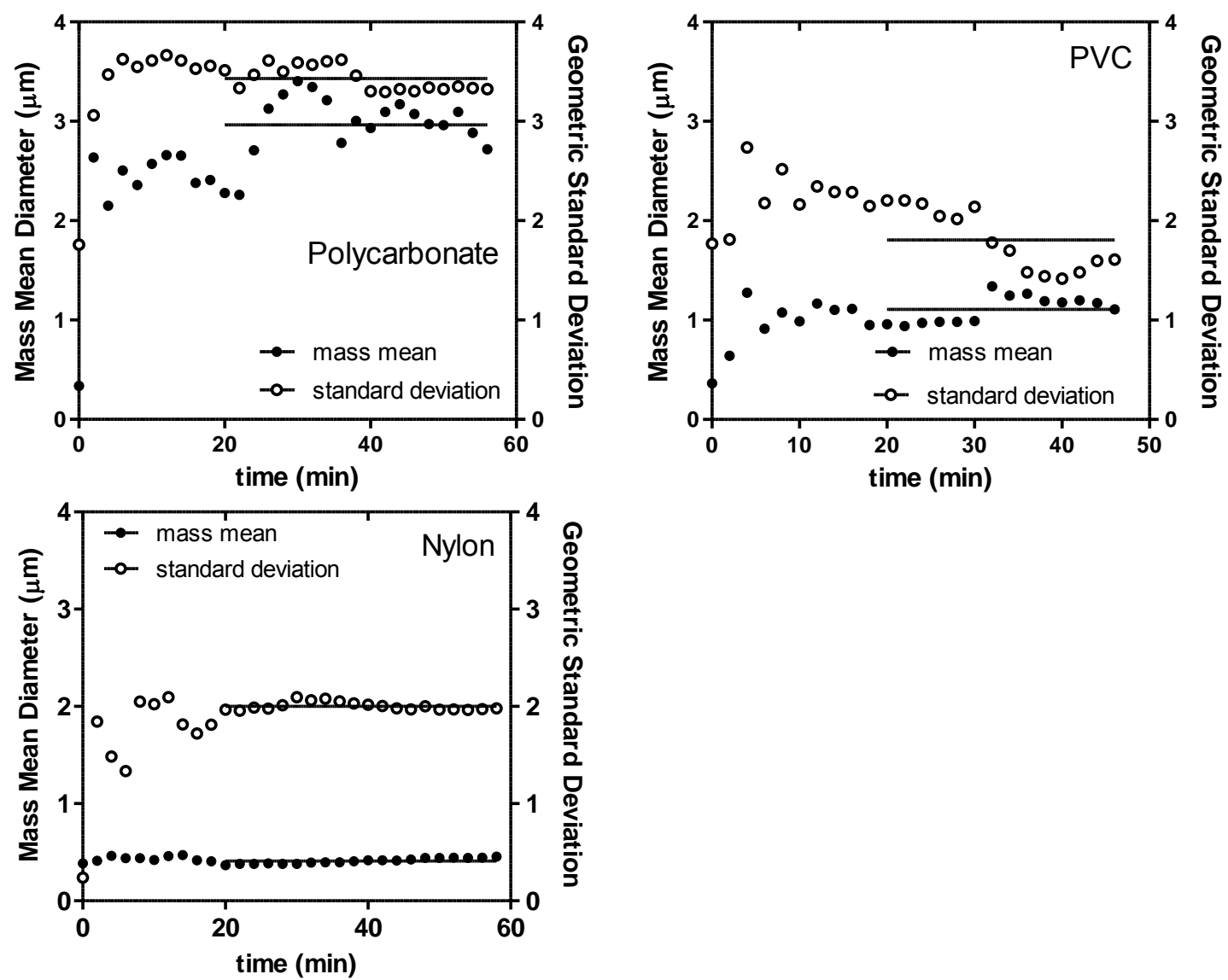

Fig. 9. Particle size statistics histories for polycarbonate, PVC and nylon smoke. 


\section{Measurements in Large-Scale Test Enclosure}

Previously, fire tests were conducted in a large test enclosure $\left(1200 \mathrm{~m}^{3}\right)$ to develop smoke concentration and deposition data from several materials (work conducted by Nam and summarized in Ref. [10]). Figure 10 shows a schematic of the test enclosure with the measurement locations. Seven tests were conducted using PMMA, polypropylene, polystyrene and polyethylene as fire sources as shown in Table 1.

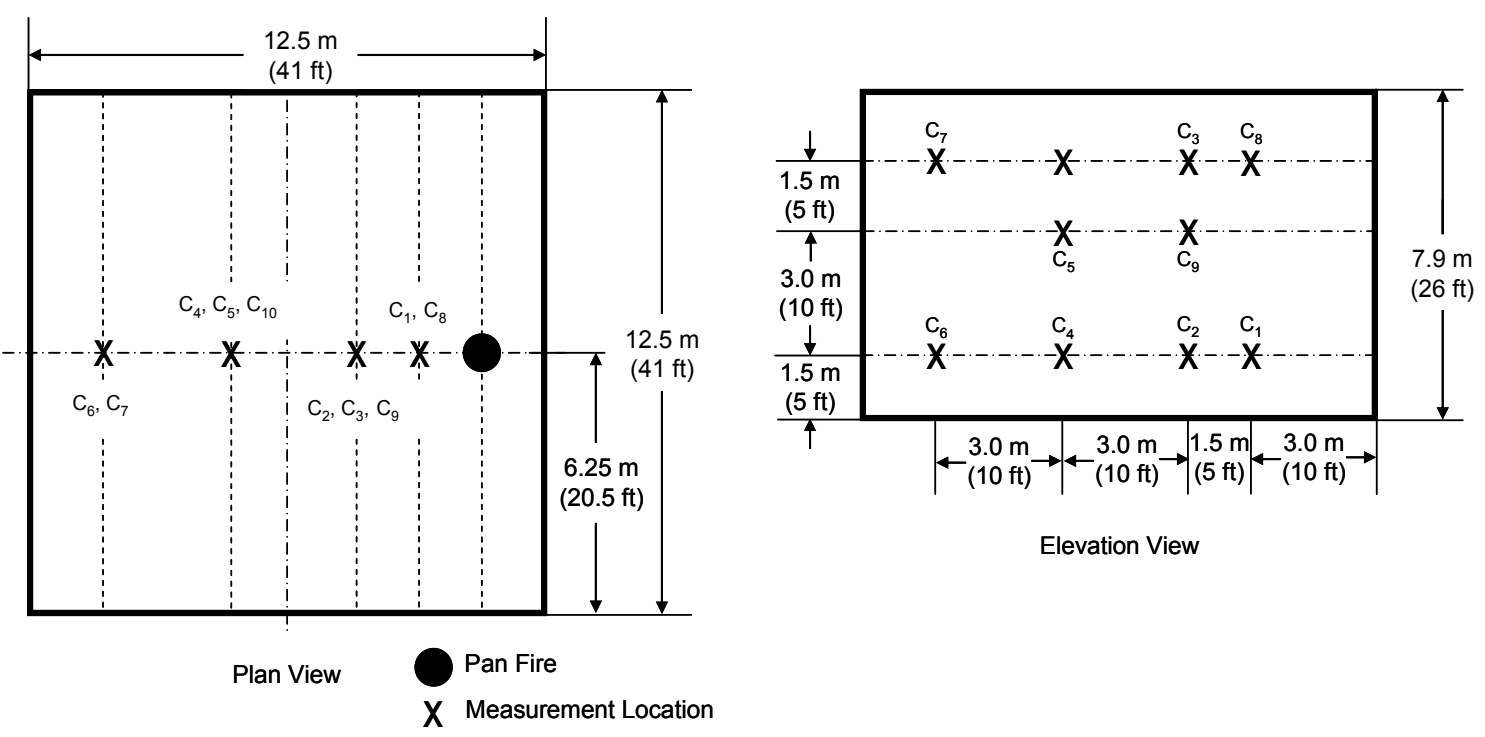

Fig. 10. Schematic of measurement locations for large-scale test enclosure (from Ref. [10]).

Table 1. Test matrix for large-scale enclosure fire tests (from Ref. [10]).

\begin{tabular}{|c|c|c|c|}
\hline Test number & Fuel material & Fuel mass (kg) & Test duration (min) \\
\hline 1 & PMMA & 13.88 & 121 \\
\hline 2 & Polypropylene & 4.87 & 158 \\
\hline 3 & Polystyrene & 2.78 & 140 \\
\hline 4 & Polyethylene & 4.82 & 175 \\
\hline 5 & Polypropylene & 4.82 & 155 \\
\hline 6 & Polypropylene & 4.83 & 42 \\
\hline 7 & Polypropylene & 4.87 & 45 \\
\hline
\end{tabular}

The WPS measurements were made at approximately mid-height in the test enclosure $(4.5 \mathrm{~m})$ and $6.0 \mathrm{~m}$ from the fire source, i.e., location $C_{5}$ as shown in Fig. 10. Figure 11 plots previously unanalyzed test data for the particle size statistics history from Tests 1-7. The particle size is given, as previously shown for the SEC data, as the mass mean aerodynamic equivalent diameter, $\vec{d}_{a}$. Also included for each plot in the figure is the geometric standard deviation, $\sigma_{g}$, for each five minute time slice plotted on the right ordinate. The straight lines in the plots for PMMA and polyethylene represent average values after 20 min from the initiation of the smoke sampling, with a $\vec{d}_{a}$ of 2.3 and $1.0 \mu \mathrm{m}$, respectively.

The polypropylene data are shown with error bars representing the data from all four of the conducted tests (Tests 2, 5-7 as shown in Table 1). The straight line representing the average value $\left(\vec{d}_{a}\right.$ of $\left.1.2 \mu \mathrm{m}\right)$ for polypropylene in the upper right-hand plot in Fig. 11 is after $40 \mathrm{~min}$ from sampling initiation. $\sigma_{g}$ remained fairly constant for PMMA, polyethylene and polypropylene with average values of 4.4, 2.5 and 2.0, respectively. The data for polystyrene in the figure show a clear trend towards particle growth during the entire test period, with a corresponding narrowing of the distribution width, most likely associated with particle agglomeration. $\vec{d}_{a}$ and $\sigma_{g}$ were about $2.4 \mu \mathrm{m}$ and 2.1, respectively, during the later stages of the test for polystyrene. 

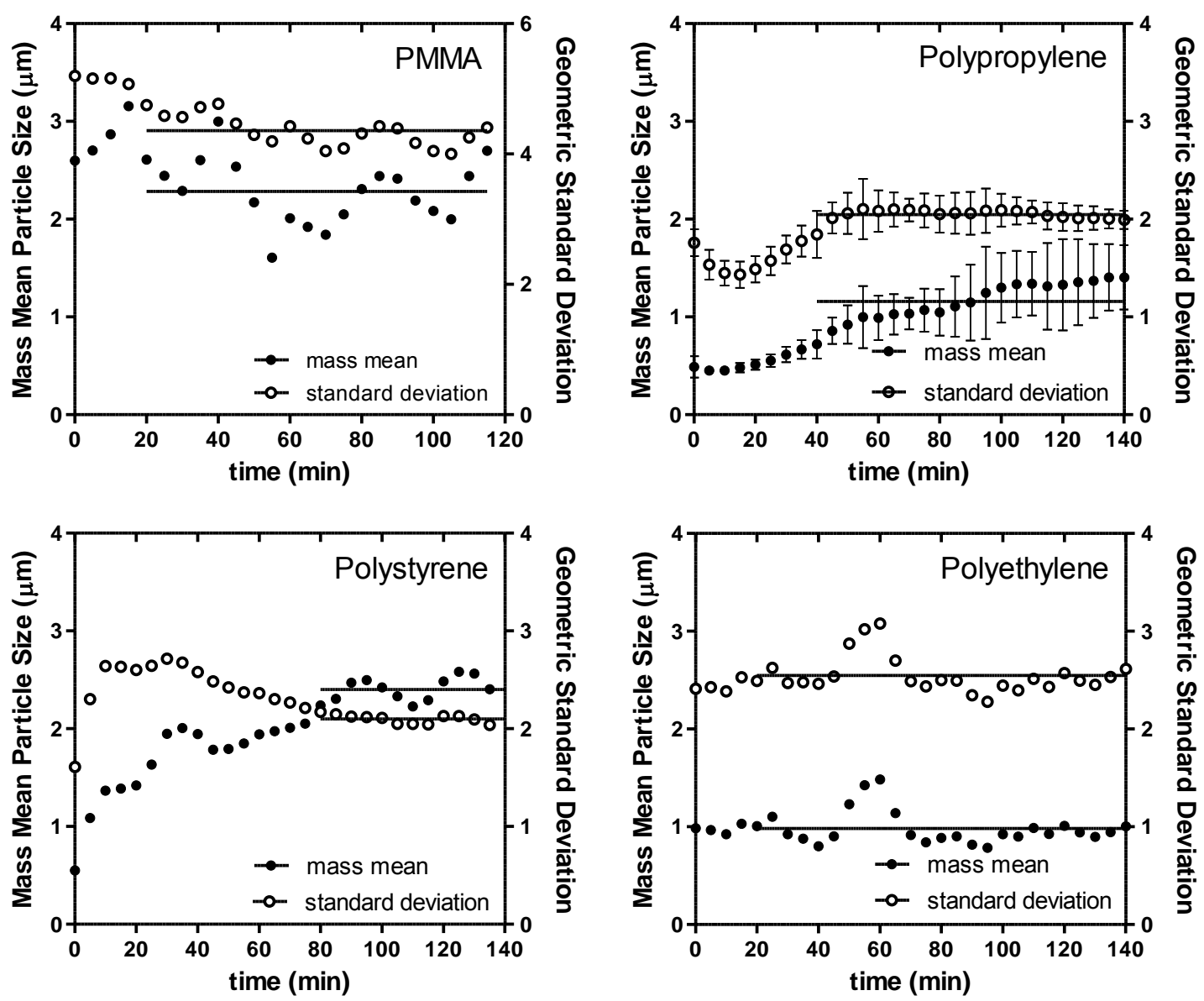

Fig. 11. Particle size statistics histories for PMMA, polypropylene, polystyrene and polyethylene smoke.

\section{SUMMARY DISCUSSION}

Smoke deposition characterization tests, conducted using a tube furnace/smoke exposure chamber apparatus, revealed that the deposition data could be represented by a complementary function determined only by the curve fit to the decay data. Two complementary functions were defined such that their sum should always yield a constant, i.e., a value of one. The decay process appears to be a two-phase exponential decay, where the overall process can be represented by the sum of a short relatively fast decay stage and a longer relatively slow decay stage. Table 2 provides a summary of the smoke deposition results for the three target materials - polycarbonate, PVC and nylon. In the table, the measured smoke deposition velocity, $V_{T S}$, together with the average particle aerodynamic mass mean diameter, $\vec{d}_{a}$, (with the associated geometric standard deviation, $\sigma_{g}$ ) are reported from smoke sedimentation experiments using a $1.0 \mathrm{~m}^{3}$ smoke exposure chamber. In addition, results reported in previous work [10] for PMMA, polypropylene, polystyrene and polyethylene deposition velocities are given from fire tests in a large-scale $1200 \mathrm{~m}^{3}$ enclosure. Previously unanalyzed particle size statistics associated with these fire tests are also given in Table 2. It is particularly interesting to note that the measured deposition velocities are quite similar for the two dramatically different scale experiments. This result is most likely due to both sets of experiments having the same dominant settling mechanism (i.e., gravimetric) for smoke deposition, even though the smoke exposure chamber was essentially a simulated quiescent deposition environment while the large enclosure employed much larger real-scale fire sources. 
Table 2. Summary results for smoke deposition.

\begin{tabular}{|c|c|c|c|c|c|}
\hline \multirow[t]{2}{*}{ Material } & \multicolumn{2}{|c|}{$\begin{array}{c}\text { Deposition velocity } \\
\left(10^{-4} \mathrm{~m} / \mathrm{s}\right)\end{array}$} & \multicolumn{2}{|c|}{$\begin{array}{c}\text { Aerodynamic mass Mean } \\
\text { diameter }(\mu \mathrm{m})\end{array}$} & \multirow{2}{*}{$\begin{array}{c}\text { Geometric standard } \\
\text { deviation }\end{array}$} \\
\hline & Measured & Calculated & Measured & Calculated & \\
\hline Polycarbonate & 1.9 & 2.9 & 3.0 & 2.4 & 3.4 \\
\hline PVC & 1.4 & 0.5 & 1.1 & 2.0 & 1.8 \\
\hline Nylon & 2.5 & 0.1 & 0.4 & 2.4 & 2.0 \\
\hline Polypropylene & 1.3 & 0.7 & 1.2 & 2.0 & 2.0 \\
\hline PMMA & 1.9 & 1.7 & 2.3 & 2.4 & 4.4 \\
\hline Polystyrene & 7.3 & 1.8 & 2.4 & 4.7 & 2.1 \\
\hline Polyethylene & 1.2 & 0.4 & 1.0 & 1.8 & 2.5 \\
\hline
\end{tabular}

The calculated deposition velocities are determined through the application of Stokes' law as given by Eq. 10 with a correction for particle slip [Eq. 3] using the measured $\vec{d}_{a}$ value for each material. Similarly, the calculated $\vec{d}_{a}$ is determined from the measured values of $V_{T S}$ for each material. As shown in the table, a not unexpected result is that the deposition velocities tend to be under-predicted. This is most likely due to cloud settling for these low particle Reynolds number [see Eq. 1] flows, where the smoke concentration is sufficient to move the smoke layer as an entity at a higher velocity than the individual particles [5]. Consequently, a diameter representative of the cloud rather than $\vec{d}_{a}$ would provide a closer prediction to the calculated results. Nevertheless, the agreement for $V_{T S}$ is reasonable, within a factor of 2 to 3 (with notable exceptions of nylon and polystyrene).

\section{REFERENCES}

[1] Hinds, W.C., Aerosol Technology - Properties, Behavior, and Measurement of Airborne Particles, $2^{\text {nd }}$ edition, John Wiley \& Sons, Inc., New York, 1999.

[2] Cunningham, E., (1910) On the Velocity of Steady Fall of Spherical Particles through Fluid Medium, Proceedings of the Royal Society A, 83:357-365, http://dx.doi.org/10.1098/rspa.1910.0024.

[3] Friedlander, S.K., and Johnstone, H.F., (1957) Deposition of Suspended Particles from Turbulent Gas Streams, Industrial and Engineering Chemistry, 49:1151-1156, http://dx.doi.org/10.1021/ie50571a039.

[4] Einstein, A., (1905) On the Kinetic Molecular Theory of Thermal Movements of Particles Suspended in a Quiescent Fluid, Annalen der Physik, 17:549, http://dx.doi.org/10.1002/andp.19053220806.

[5] Hinds, W.C., Ashley, A., Kennedy, N.J., and Bucknam, P., (2002) Conditions for Cloud Settling and Rayleigh-Taylor Instability, Aerosol Science and Technology, 36:1128-1138, http://dx.doi.org/10.1080/02786820290108449.

[6] Newman, J.S., and Steciak, J., (1987) Characterization of Particulates from Diffusion Flames, Combustion and Flame, 67:55-64, http://dx.doi.org/10.1016/0010-2180(87)90013-7.

[7] ASTM E2058, "Standard Test Methods for Measurement of Synthetic Polymer Material Flammability Using a Fire Propagation Apparatus (FPA)," ASTM International, West Conshohocken, PA, DOI: 10.1520/E2058-09, 2009.

[8] Tewarson, A., "Generation of Heat and Gaseous, Liquid, and Solid Products in Fires," The SFPE Handbook of Fire Protection Engineering, $4^{\text {th }}$ edition, NFPA, Quincy, MA, 2008, p. 3/109.

[9] Liu, B.Y.H, Romay, F.J., Dick, W.D., Woo, K-S, and Chiruta, M., (2010) A Wide-Range Particle Spectrometer for Aerosol Measurement from $0.010 \mu \mathrm{m}$ to $10 \mu \mathrm{m}$," Aerosol and Air Quality Research, 10:125-139. 
[10] Newman, J.S., Su, P., Yee, G.G., Jamison, K.L.T., and Chivukula, S., "Strategic Smoke Damage Program: Development of Smoke Damage Functions for the Semiconductor Industry," FM Global Technical Report, Project ID 0003038685, September 2010. 